\title{
Casos clínicos y cine en la docencia del máster en neurocontrol motor
}

\section{Susana Collado Vázquez ${ }^{a}$, Jesús María Carrillo}

${ }^{a}$ Departamento de Fisioterapia, Terapia Ocupacional, Rehabilitación y Medicina Física. Facultad de Ciencias de la Salud. Universidad Rey Juan Carlos. susana.collado@urjc.es bepartamento de Personalidad, evaluación y Psicología clínica. Facultad de Psicología de la Universidad Complutense de Madrid.jmcarril@psi.ucm.es

\begin{abstract}
Since ancient times have been used clinical cases in degrees of Health Sciences, both undergraduate and graduate students to put to real cases found in the clinical practice of degrees that course or in their professional lives. Clinical cases help students to reflect and put in real situations.

The film has multiple stories of all kinds and various genres such as drama, science fiction, western, comedy, etc. Often health and disease and the various professions Health Sciences d ela are present and therefore there is a material used in teaching. Many patients fiction can become clinical case studies in the classroom, and this methodology is used in various subject of Health Sciences and Ethics in research and clinical practice based on evidence of the Master in NeuroControl Motor University King Juan Carlos and have served to analyze clinical cases related to ethics or with various neurological diseases.
\end{abstract}

Keywords:Cinema, Case reports, teaching, educational innovation, Neurology 


\begin{abstract}
Resumen
Desde antiguo se han empleado casos clínicos en titulaciones de Ciencias de la salud tanto de grado como de postgrado, para poner a los estudiantes ante casos reales que encontrarán en la prácticas clínicas de las titulaciones que cursen o en su vida profesional. Los casos clínicos ayudan a los estudiantes a reflexionar y a ponerse en situaciones reales.

El cine cuenta múltiples historias de todo tipo y de diversos géneros como drama, ciencia ficción, western, comedia, etc. Con frecuencia la salud y la enfermedad y las diversas profesiones de Ciencias d ela Salud están presentes y por tanto existe mucho material que emplear en docencia. Son muchos los pacientes de ficción que pueden convertirse en casos clínicos para analizar en el aula, y esta metodología es utilizada en diversas asignatura de Ciencias de la Salud como Ética en investigación y Práctica clínica basada en la evidencia del Máster en Neurocontrol Motor de la Universidad Rey Juan Carlos y que han servido para analizar casos clínicos relacionados con la ética o con diversas enfermedades neurológicas.
\end{abstract}

Palabras clave: Cine, Casos clínicos, docencia, innovación educativa, Neurología

\title{
Introducción
}

Desde antiguo se ha empleado el estudio de casos en diversas carreras como Derecho y Medicina, por tanto, es una práctica habitual y útil en Ciencias de la salud el empleo de casos clínicos, tanto en grado como en postgrado.

La utilización de casos acerca a los estudiantes a su práctica clínica colocándolos en contextos y situaciones reales, próximas a lo que será su práctica profesional y les ayuda a integrar conocimientos de diversas asignaturas, reflexionar, optar por unas opciones diagnósticas, pronósticas, terapéuticas, realizar una valoración ética, buscar bibliografía que apoye sus decisiones, entre otros aspectos (1).

Desde los inicios del séptimo arte el cine se ha interesado por la salud y la enfermedad, los profesionales sanitarios, los avances médicos, la vivencia de pacientes, familiares y cuidadores, métodos diagnósticos y terapéuticos, entre otros aspectos, esto hace que contemos con abundante material fílmico que utilizar en el aula con fines didácticos o divulgativos (1-3). 
Existen muchas historias en las que los pacientes de diversas afecciones están muy presentes como protagonsitas, prácticamente todas las patologías han sido reflejadas en el cine, y si se emplean en docencia casos clínicos de pacientes reales como metodología educativa ¿Por qué no emplear casos clínicos de pacientes de ficción como metodología docente para alumnos de Ciencias de la Salud?

En algunos casos se ha hecho la crítica de que las películas son historias inventadas y que no siempre muestran de forma correcta las patologías, su abordaje, la actitud de los profesionales, los métodos diagnósticos o terapéuticos, pero esto, no tiene que constituir un obstáculo para la utilización de personajes de películas a modo de casos clínicos, ya que los estudiantes, con los conocimientos previamente adquiridos, deberán saber distinguir lo real de lo erróneamente representado, lo que requiere una importante capacidad de observación, reflexión, análisis crítico e integración de conocimientos previamente aprendidos (1).

En relación con las afecciones neurológicas son muchas las películas que muestran diversos cuadros patológicos con diferente enfoque y más o menos ajustados a la realidad, y asimismo se han publicado numerosos trabajos científicos sobre la representación de la enfermedad neurológica en el cine: demencia, coma, amnesia o esclerosis múltiple por poner algunos ejemplos (4-8).

En el Máster Universitario en Neurocontrol Motor de la Universidad Rey Juan Carlos se han empleado diversas películas en las que aparece algún paciente, como casos clínicos, para trabajar en el aula y ayudar a los estudiantes en la adquisición de competencias específicas y transversales. Se ha empleado en diversas asignaturas pero nos centraremos en la aplicación en la asignatura de Práctica clínica basada en la evidencia.

\section{Objetivos}

Trabajar mediante casos clínicos de cine para colocar al alumno en situaciones similares a las que encontrará en su práctica profesional.

Contribuir en la adquisición de competencias específicas y transversales como observación, capacidad de reflexión, de solución de problemas nuevos, crítica, trabajo autónomo y colaborativo, comunicación oral y escrita.

\section{Desarrollo de la innovación}

La asignatura práctica clínica basada en la evidencia (3 créditos ECTS) del Máster Universitario en Neurocontrol Motor, se centra en el estudio de las enfermedades neurológicas buscando las mejores evidencias en relación con los abordajes terapéuticos.

Se analizan patologías como las demencias, parálisis cerebral, tumoraciones neurológicas, accidente cerebrovascular, lesión medular, enfermedad de Parkinson, esclerosis múltiple o esclerosis lateral amiotrófica, entre otros cuadros.

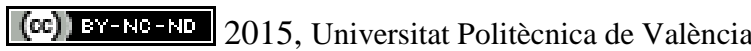


Se seleccionaron diversos fragmentos fílmicos de películas como Hombres e Intocable (lesión medular), Iris, Lejos de ella, Arrugas, My name is Lisa (Enfermedad de Alzheimer), Amarga victoria (tumor del sistema nervioso), o Mi pie izquierdo, Las llaves de casa, Gaby, una historia verdadera (parálisis cerebral). (Tabla 1).

Tabla 1. Selección de películas en las que aparecen enfermedades neurológicas

\begin{tabular}{|l|l|}
\hline \multicolumn{1}{|c|}{ Título de la película } & \multicolumn{1}{c|}{ Enfermedad o lesión } \\
\hline A propósito de Henry & Daño cerebral \\
\hline Sublime obsesión & Tumor del sistema nervioso y ceguera \\
\hline El orgullo de los Yankees & Esclerosis lateral amiotrófica \\
\hline La escafandra y la mariposa & Síndrome de cautiverio \\
\hline Al rojo vivo & Cefaleas \\
\hline El regreso & Lesión medular \\
\hline Nacido el 4 de julio & Lesión medular \\
\hline Go now & Esclerosis múltiple \\
\hline Bailo por dentro & Parálisis cerebral \\
\hline Los descendientes & Coma, enfermedad de Alzheimer \\
\hline Aurora borealis & Enfermedad de Parkinson \\
\hline Amor y otras drogas & Enfermedad de Parkinson \\
\hline
\end{tabular}

Se elaboró una ficha de cada película y se proyectaron los fragmentos fílmicos seleccionados y se pidió a los estudiantes que por pequeños grupos analizaran la información facilitada y las escenas proyectadas, indicando si las pruebas diagnósticas o tratamientos aplicados eran correctos y estaban bien representados en el filme o qué protocolos habrían seguido ellos en ese caso en concreto. Asimismo analizar el contexto histórico del filme para comprender mejor las técnicas de diagnóstico y tratamiento y valorar si eran las que habitualmente se empleaban en la época histórica que se representa en la película.

Tras el trabajo en equipo se realizó un debate para poner en común lo reflexionado en cada grupo y discutir las opciones de evaluación y tratamiento propuestas por cada grupo de trabajo. 


\section{Resultados}

En esta actividad participan todos los alumnos del Máster en Neurocontrol Motor de la asignatura Práctica clínica basada en la evidencia, los cuales realizan un trabajo individual, analizando el material facilitado, un trabajo en pequeños grupos y un debate general.

Con esta metodología se produce un alto nivel de implicación y participación de los alumnos que manifiestan un alto grado de satisfacción pues según comentan les ayuda a observar, reflexionar, trabajar en grupo, resolver problemas nuevos y acercarse a contextos similares a los profesionales, es decir, es una metodología adecuda para la adquisición de competencias específicas y transversales, para repasar conceptos aprendidos e integrar información de diversas asignaturas de la titulación, para poder resolver el problema propuesto.

El 100\% de los alumnos encuestados valoró la actividad con una puntuación entre 8-10, como sugerencia apuntaron la utilización de películas más recientes, inidcaron que les gustarían actividades similares en otras asignaturas por su amenidad y porque de forma dinámica les permitía aprender, reflexionar y enfrentarse a situaciones que requieren la resolución de problemas cercanos a su práctica profesional. Asimismo valoraron favorablemente el trabajo en equipo que les permitió conocer mejor a compañeros con los que no tenían mucho trato, y les enriqueció por el intercambio de opiniones y conocimientos, y el debate les sirvió para valorar distintas opciones en la evaluación y tratamiento de los pacientes.

Entre los fragmentos o cortos más valorados se encuentran: My name is Lisa, Mi pie izquierdo, Arrugas o Las llaves de casa.

Asimismo los estudiantes sugirieron títulos como me llaman Radio, Forrest Gump, Las sesiones, y en este último curso: Siempre Alice o la Teoría del Todo, entre otro títulos. Otra sugerencia fue presentar además del caso clínico de la película un caso clínico real similar, o apoyar la actividad con vídeos reales.

\section{Conclusiones}

La metodología utilizada se muestra útil para fomentar competencias específicas y transversales (reflexión, comunicación oral y escrita, resolución de problemas nuevos, observación y reflexión crítica).

Esta actividad ha fomentado la participación e implicación de los estudiantes, que suelen estar muy motivados por encontrarse en contextos similares a su práctica profesional.

(cc)) EY-NC-ND 2015, Universitat Politècnica de València

Congreso In-Red (2015) 
Además de la participación en el caso clínico los alumnos han aportado sugerencias para mejorar esta actividad, lo que muestra el interés, participación e implicación d elos alumnos.

\section{Referencias}

1. Collado-Vázquez S, Carrillo JM. Cine y Ciencias de la Salud. Aplicaciones docentes. Madrid:Dykinson;2013.

2. García Sánchez JE, Garcí sanchez E. Medicina, Cine y Educación. Rev Med Cine 2008;4(2):39-40.

3. García JE, Trujillano I, García E. Medicina y cine ¿Por qué? Rev Med cine 2005;1 (1):1-2.

4. Collado Vázquez S, Cano de la Cuerda R, Jiménez Antona C. Deficiencia, discapaidad, neurología y cine. Rev Neurol 2010;51(12):757-763.

5. Karenberg A. Multiple sclerosis on screen: from disaster to coping. Multiple sclerosis 2008;14:530-40.

6. Wijdicks EFM, Wijdicks CA. The portrayal of coma in contemporary motion pictures. Neurology 2006;66:1300-3

7. Segers K. Degenerative dementias and their medical care in the movies. BMJ 2004;21:55-9.

8. Baxendale S. Memories aren 't made of this: amnesia at the movies. BMJ 2004;329:1480-3 